\title{
Depressed BCG Vaccination Scar on the Arm Successfully Treated by a Combination of Fractional 2,940-nm Erbium:Yttrium Aluminum Garnet Laser and Subcision
}

Young Koo Kim ${ }^{1}$

Sang Ju Lee ${ }^{1}$

Miri Kim²

Ji Eun Kim

Sang Eun Lee ${ }^{3}$

\footnotetext{
${ }^{1}$ Yonsei Star Skin \& Laser Clinic, Seoul, Korea

${ }^{2}$ Department of Dermatology, Yeouido St. Mary's Hospital, College of Medicine, The Catholic University of Korea, Seoul, Korea

${ }^{3}$ Department of Dermatology, Gangnam Severance Hospital, Cutaneous Biology Research Institute, Yonsei University College of Medicine, Seoul, Korea
}

\begin{abstract}
Bacille Calmette-Guérin vaccine (BCG) vaccination is a common cause of depressed scar on the body. Nonfacial depressed scars are relatively more difficult to treat without avoiding any side effects when compared with facial scars, because nonfacial tissues have greater cutaneous thickness and poor would healing. Ablative fractional resurfacing (AFR) systems have proven to be effective for atrophic scars that result from acne and trauma. The efficacy of AFR using carbon dioxide $\left(\mathrm{CO}_{2}\right)$ laser has been well documented for facial atrophic scars; but for nonfacial depressed scars on the body, the effect of AFR using $\mathrm{CO}_{2}$ or $2,940-\mathrm{nm}$ erbium-doped yttrium aluminum garnet (Er:YAG) laser has not been well documented. Subcision is another method used to manage depressed scars. We treated depressed BCG vaccination scar on the arm with a combination of fractional 2,940-nm Er:YAG laser and subcision. We showed significant improvements without serious side effects, such as prolonged erythema or hyperpigmentation.
\end{abstract}

\section{Key words}

Depressed scar; Arm; Fractional 2,940-nm Er:YAG laser; Subcision 


\section{INTRODUCTION}

The location of the cutaneous injury is one of the factors affecting the type and severity of scar. Compared to facial scars, scarring on the nonfacial areas in the body generally have tendency to hypertrophy due to the relatively high-tension environment created by the large muscles, the increased pressure or shear over sites. 'However, on the body region, atrophic or depressed scars can also occur following various traumatic or surgical injuries.

Ablative fractional resurfacing (AFR) systems including fractional carbon dioxide $\left(\mathrm{CO}_{2}\right)$ and fractional erbiumdoped yttrium aluminum garnet (Er:YAG) lasers are demonstrated to be effective in the treatment of atrophic facial scars by producing microscopic ablation zones which induce dermal remodeling, collagen synthesis, and tissue tightening. ${ }^{2,3}$ However, the use of AFR for treating nonfacial atrophic or depressed scars on the body was not as well documented. There have been several case series and noncontrolled studies which report successful treatment of atrophic scars on the body with fractional $\mathrm{CO}_{2}$ laser ${ }^{4-6}$ but there are few reports on the use of fractional Er:YAG lasers for the depressed scars on nonfacial body regions.

Depressed scars can be caused by deep inflammatory reaction which leads to the destruction of connective tissue with dermal atrophy as well as deep fibrosis. Therefore, subcision is also an effective modality to improve the depressed scars by releasing scar surfaces from underlying attachments. ${ }^{7}$ In this report, we present a case of depressed BCG vaccination scar on the arm successfully treated by combination of fractional 2,940-nm Er:YAG laser and subcision.

\section{CASE REPORT}

A 13-year-old female patient presented with BCG vaccination scar on the left upper arm and desired cosmetic treatment of the scars. Physical examination revealed 18 tiny round dots on her upper arm and each dot was depressed and reached a diameter of approximately 4-6 mm (Fig. 1A). She had no other treatments prior to visiting our clinic. The depressed scars on her arm were treated with combination of 6 sessions of fractional $a b-$ lative 2,940-nm Er:YAG laser (JOULE, Profractional-XC mode, Sciton, Inc., Palo Alto, CA) treatments at 2 month intervals and two times of subcisions. Scars were photographed at baseline and after each treatment using a digital camera. Before the laser treatment, 5\% lidocaine cream (EMLA, AstraZeneca, London, UK) was applied with occlusion. Fractional Er:YAG laser treatments were performed using a spot size $400 \mu \mathrm{m}$. One or two passes of treatments were performed with densities of $5.5 \%$ skin surface area coverage. Coagulation level 0 was used, as depth range from 500-1500 $\mu \mathrm{m}$. When the punctate bleeding during the procedure was observed, energy densities were decreased to reduce posttreatment erythema. Ice-packing was done after the treatment to minimize
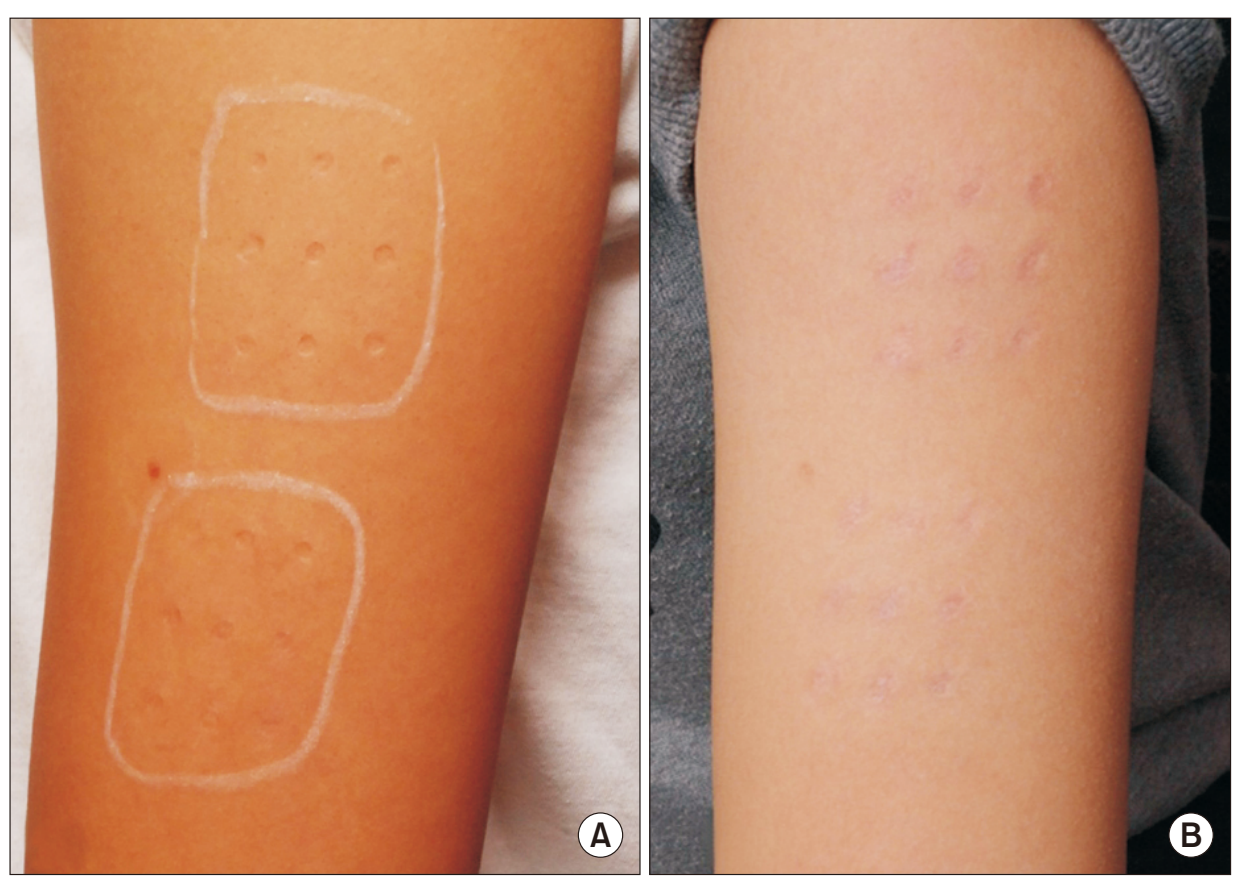

Fig. 1. BCG vaccination-induced depressed scar located the arm (A). Two months after the combination of fractional 2,940-nm erbium:yttrium aluminum garnet (Er:YAG) laser treatment and subcision (B). 
heating sensation and pain. After each session of the laser treatment, immediate postprocedure erythema and edema was observed, but when the patient returned for evaluation 2 months after each laser treatment, erythema disappeared spontaneously. No treatment-induced dyschromia was observed. After 4th and 5th session of laser treatment, subcision was performed. 2 months later, 6th session of fractional Er:YAG laser treatment was performed. Improvement in atrophy, skin texture, and overall appearance started to be noted after four sessions of fractional Er:YAG laser treatments, and subsequent two times of subcision and two sessions of fractional Er:YAG laser treatments resulted in significant improvement in all 18 depressed scars (Fig. 1B).

\section{DISCUSSION}

Nonfacial atrophic or depressed scars located in the body present unique therapeutic challenges due to the greater cutaneous thickness and the potential of poor wound healing compared to the facial scars. BCG vaccination is a common cause of nonfacial depressed scarring which has been reported to be formed in 47.2 to $100 \%$ of BCG vaccinated newborns after 12 weeks of vaccination. $^{8}$

AFR has been reported to be a safe and effective method for treating facial atrophic scars, in contrast, evidence for the effectiveness of AFR on nonfacial atrophic or depressed scars is lacking. To treat nonfacial scars located in body regions of greater cutaneous thickness using AFR systems, a higher fluence leading to a greater ablation and coagulation depth may offer a better clinical outcome theoretically. However, in dark-skinned individuals, laserinduced prolonged erythema and dispigmentation may frequently occur with the AFR systems, especially in the setting of high energy. In fact, a recent randomized controlled study on fractional $\mathrm{CO}_{2}$ laser for various scar type noted that side effects were frequently reported when the scars were located on the torso and extremities. ${ }^{3}$

Compared to 10,600-nm CO 2 AFR laser, Er:YAG AFR laser at a wavelength of 2,940 $\mathrm{nm}$ can provide less ablation and coagulation depth, so it has been postulated that Er:YAG AFR laser may be less effective for atrophic scars. However, a recent study which compared the two AFR systems in resurfacing of atrophic acne scars in Asians reported that fractional $\mathrm{Er}: Y A G$ and $\mathrm{CO}_{2}$ lasers offered comparable clinical outcomes, but fractional $\mathrm{CO}_{2}$ laser was associated with greater treatment-associated side effects including postinflammatory hyperpigmentation, thereby suggesting that fractional Er:YAG laser may be a better choice for treating atrophic acne scars in Asian patients than the fractional $\mathrm{CO}_{2}$ laser. ${ }^{9}$ Taking together with the potential risks and benefits, we used fractional Er:YAG laser for BCG vaccination-induced depressed scar on the body. After 4 sessions of fractional Er:YAG laser treatment, skin texture, depression, and overall appearance were significantly improved in our case and postoperative erythema and pigmentation not lasted for two months after the treatment. In our experience, we suggest that Er:YAG AFR laser can be safe and effective in treating depressed scar located on the body in dark skinned individual.

BCG vaccination-induced depressed scar on the arm is deep and fibrotic. Beside the trauma and inflammationinduced dermal atrophy, fibrous tissue formation with abnormal fibrous anchoring is also another aetiology for depressed scar. Subcision is a simple surgical technique used to manage depressed scars by releasing fibrous attachments beneath the scar and inducing the formation of connective tissues through normal physiological healing. Therefore, combined treatment of subcision with AFR may provide additive improvement for this type of depressed scar. Our results showed that combining ablative Er:YAG fractional laser and subcision improve the clinical appearance of depressed scar on the nonfacial body region without significant adverse effects associated with AFR treatment such as prolonged erythema and hyperpigmentation. Although, the treatment protocols, efficacy, and safety should be established through further controlled clinical trials that involve a large number of patients, we concluded that the combination of fractional Er:YAG laser and subcision can be safe and effective treatment for depressed scars on the body in Asian patients.

\section{REFERENCES}

1. Keaney TC. Prevention and treatment of nonfacial scars. Semin Cutan Med Surg 2015;34:153-7.

2. Hedelund L, Haak CS, Togsverd-Bo K, Bogh MK, Bjerring P, Haedersdal M. Fractional CO2 laser resurfacing for atrophic acne scars: a randomized controlled trial with blinded response evaluation. Lasers Surg Med 2012;44:447-52.

3. van Drooge AM, Vrijman C, van der Veen W, Wolkerstorfer A. A randomized controlled pilot study on ablative fractional CO2 laser for consecutive patients presenting with various scar types. Dermatol Surg 2015;41:371-7.

4. Shumaker PR, Kwan JM, Landers JT, Uebelhoer NS. Functional improvements in traumatic scars and scar contractures using an ablative fractional laser protocol. J 
Trauma Acute Care Surg 2012;73(2 Suppl 1):S116-21.

5. Haedersdal M. Fractional ablative CO(2) laser resurfacing improves a thermal burn scar. J Eur Acad Dermatol Venereol 2009;23:1340-1.

6. Weiss ET, Chapas A, Brightman L, Hunzeker C, Hale EK, Karen JK, et al. Successful treatment of atrophic postoperative and traumatic scarring with carbon dioxide ablative fractional resurfacing: quantitative volumetric scar improvement. Arch Dermatol 2010;146:133-40.
7. Orentreich DS, Orentreich N. Subcutaneous incisionless (subcision) surgery for the correction of depressed scars and wrinkles. Dermatol Surg 1995;21:543-9.

8. Faridi MM, Krishnamurthy S. Abortive reaction and time of scar formation after BCG vaccination. Vaccine 2008;26:289-90.

9. Manuskiatti W, lamphonrat T, Wanitphakdeedecha R, Eimpunth $\mathrm{S}$. Comparison of fractional erbium-doped yttrium aluminum garnet and carbon dioxide lasers in resurfacing of atrophic acne scars in Asians. Dermatol Surg 2013;39:111-20. 